(Paper No. 3037.)

\title{
"Fissures in Masonry Dams."
}

By Ernest Prescot Hill, M. Inst. C.I.

THE destructive effect, under certain circumstances, of a horizontal fissure occurring on the water-face of a masonry dam, as exemplified by the failure of the Bouzey dam, has been pointed out by Professor W. C. Unwin. ${ }^{1}$ The object of the present communication is to examine the circumstances under which fissures may be formed, and to obtain expressions in general terms which show the effect of a fissure when once formed.

It is necessary, first, to distinguish between two classes of fissures. The first class comprises those due to bad workmanship

Fig. 1.

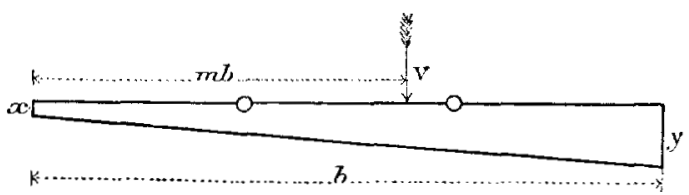

particularly when new and old work are not properly united, these terms being here used in a relative sense, and referring to the interval during suspension of work from whatever cause, either during the night, the week-end, or public holidays. The effect of the failure to unite the two surfaces (and without the greatest care this may easily occur) is to cause literally a horizontal fissure, into which the water of the reservoir will enter. In certain cases, which will be described, there is no tendency of the fissure to develop, and the effect is merely to tend to vary the stresses along the plane in question within narrow limits. In other cases such tissures may tend to develop. The second class includes fissures due to bad design; these tend to become progressive, as will be presently shown.

1 Minates of Proceedings Inst. C.E., vol. exxvi. pp. 93, 94, and Cassier's Magazine, November, 1896. 
Papers.]

In Fig. 1 are shown the vertical components of the stresses for the case when the distance of the resultant of the vertical forces acting at any particular horizontal plane, measured from the inner face of the dam, is less than two-thirds of the breadth of the dam at that plane; $V$ being the vertical component of the resultant for the unit length of dam; $b$ the breadth of the dam at the plane considered; $m b$ the distance of $\nabla$ from the inner face of the dam; $x$ the stress-intensity at the inner face; and $y$ the stress-intensity at the outer face. The ordinates when measured below the horizontal line are positive or compressive stresses; then the following conditions exist :-

$$
\begin{gathered}
\frac{1}{2} b^{2} x+\frac{2}{3} b^{2} \cdot \frac{y-x}{2}=m b \mathrm{~V} \\
b \cdot \frac{x+y}{2}=\mathrm{V}
\end{gathered}
$$

therefore

$$
\begin{aligned}
& x=\frac{2 \mathrm{~V}}{b}(2-3 m) . \\
& y=\frac{2 \mathrm{~V}}{b}(3 m-1) .
\end{aligned}
$$

Fig. 2.

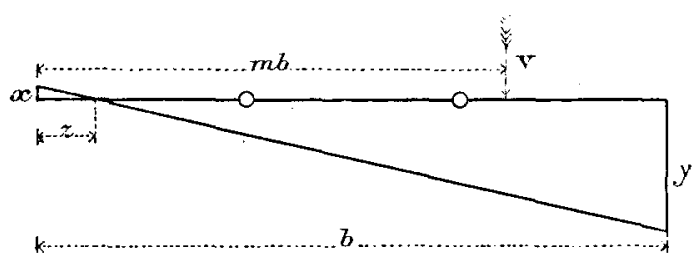

When $m=\frac{2}{3}$, then $x=0$, and $y=\frac{2 \mathrm{~V}}{b}$. When $m>\frac{2}{3}$ (a case of bad design), then $x$ is negative, and is a tensile stress. The stress diagram for this case is shown in Fig. 2.

'The relations here are:-

$$
\begin{aligned}
\frac{y}{2}(b-z)\left(\frac{2 b+z}{3}\right)-\frac{x z^{2}}{6} & =m b \mathrm{~V} \\
\frac{y}{2}(b-z)-\frac{x z}{2} & =\mathrm{V} \\
\frac{x}{z} & =\frac{y}{b-z} ;
\end{aligned}
$$


therefore

$$
\begin{aligned}
& x=-\frac{2 V}{b}(2-3 m) . . \\
& y=\frac{2 V}{b}(3 m-1) \cdot . \\
& z=\frac{b}{3} \cdot \frac{3 m-2}{2 m-1} . .
\end{aligned}
$$

With the exception of the sign of $x$, the values of $x$ and $y$ in (3) and (4) are identical with those in (1) and (2). It is clear that, in this case, if the value of the stress of which $x$ is the vertical component is greater than the strength of the masonry, a fissure will be formed. If the masonry be well bonded vertically, as well as horizontally, and if the work be of first-rate quality, the strength is very considerable. If this is not the case, the strength

Fig. 3.

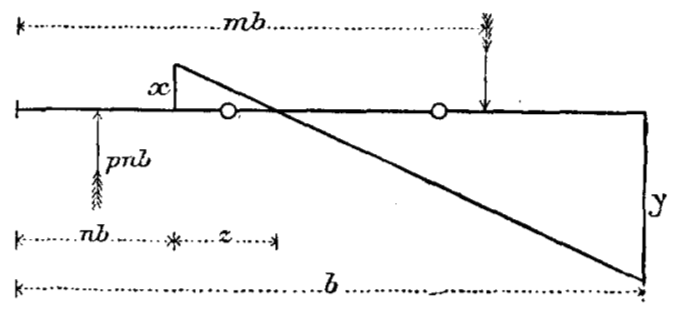

of the masonry is that of the mortar, and may be very small indeed.

If a fissure is formed in the manner indicated, another force enters into consideration, viz., the pressure of the water in the fissure. This case requires separate treatment, and the stress diagram is given in Fig. 3, where $n b$ is the depth of the fissure, $p$ the intensity of the water-pressure in the fissure, and $p n b$ the resultant water-pressure.

The relations are:-

$$
\begin{aligned}
\frac{y}{2}(b-n b-z)\left(\frac{2 b+n b+z}{3}\right)-\frac{x z}{2}\left(n b+\frac{z}{3}\right)+\frac{p n^{2} b^{2}}{2} & =m b \mathrm{~V} \\
\frac{y}{2}(b-n b-z)-\frac{x z}{2}+p n b & =\mathrm{V} \\
\frac{x}{z} & =\frac{y}{b-n b-z}
\end{aligned}
$$


therefore

$$
\begin{aligned}
& x=\frac{2 \mathrm{~V}}{b} \cdot \frac{3 m-2-n}{(1-n)^{2}}+p \frac{n(4-n)}{(1-n)^{2}} . . \\
& y=\frac{2 \mathrm{~V}}{b} \cdot \frac{3 m-1-2 n}{(1-n)^{2}}+p \frac{n / 2+n)}{(1-n)^{2}} . .
\end{aligned}
$$

If it were possible that no water should enter it, the fissure would not develop beyond the point at which $x$ (or the stress of which $x$ is the vertical component) is equal to the ultimate tensile strength of the mortar (if the mortar be in the line of least resistance); that value of $x$ is $\frac{2 \mathrm{~V}}{b} \cdot \frac{3 m-2-n}{(1-n)^{2}}$, so that, if the strength of the mortar is known, then what may be termed the initial and minimum depth of the fissure can be found. When the water enters the fissure so caused, the value of $x$ is, if the profile only of the dam be considered, greater than the strength of the mortar, and consequently the depth of the fissure is increased.

In expression (6) let $p=r \frac{\mathrm{V}}{b}$, and let $3 m-2=a$, so that $a>0$, and $<1$. Then, by differentiation,

$$
\frac{b}{\overline{\mathrm{V}}} \frac{d x}{d n}=\frac{2}{(1-n)^{3}}\{2 a+2 r-1-n(1-r)\} .
$$

Therefore $x$ decreases at an increasing rate, when $2 a+2 r<1$; increases to a maximum and then declines when $2 a+2 r=1$; increases to a maximum and then declines when $2 a+2 r>1$; increases to a maximum and then declines when $2 a+3 r=2$; and increases at an increasing rate when $2 a+3 r>2$.

The lower limit of the maximum occurs when $n=0$, and at the higher limit when $n=1$, for which value the maximum point is at an infinite distance from the line along which $n$ is measured. Between these limits the maximum point is reached when $n=\frac{2 a+2 r-1}{1-r}$. In applying these expressions to practice, it must be remembered that the values of $m$ and $r$ must be such that the initial fissure can occur. The value of $m$ which would allow such a fissure is, even in a very badly designed dam, dne to the value of $r$; and if $r$ is not sufficiently great to give a high value to $m$, no initial fissure can occur. This condition at once excludes those under which $x$ can have a diminishing value, or a maximum value at or near the lower limit. When $m$ and $r$ have values possible in practice, the maximum point has advanced so far [THE INST. C.E. VOL. CXXIX.] 
towards the higher limit of $n$, and is so far distant from the line along which $n$ is measured, that the dam will have been destroyed ling before such a point is reached, and therefore very long before that value of $n$ is reached at which, theoretically, the value of $x$ would sink below the tensile strength of the mortar. When the difference of $n$ from unity is infinitely small, the values of $x$ are, in all cases, infinitely great.

The values of $x$ and $y$ at the fissure in the Bouzey dam, are shown in Fig. 4, for every value of $n$ up to 0.9 , beyond which the values of $x$ and $y$ are too great to show. In this case $2 a+3 r>2$; in fact $2 a+3 r=2 \cdot 513$, so that the curve always rises at an increasing rate. These values hold only when the profile alone of the dam is considered, and the help given by the dam at each end

Fig. 4.

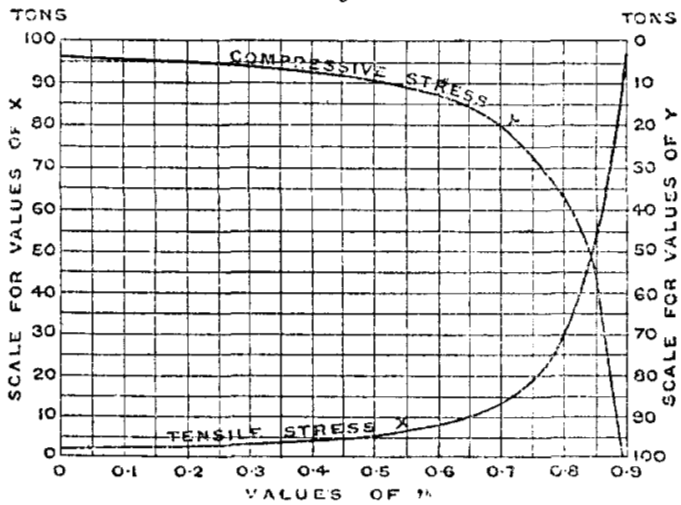

of the fissure neglected. The influence of the ends of the dam in modifying the stresses along the fissure in a transverse direction will be considered separately.

Before complete failure can occur, the overturning force must, as pointed out by Professor Unwin, be sufficient to shear through the dam in a vertical plane at each end of, and above the level of, the fissure. The supporting value of the ends is, however, very small compared with the work which would be thrown upon them by a slight variation in the strength of the mortar accumulating over a long fissure. For instance, the cross-sectional area of the Bouzey dam above the plane of fissure was about 520 square feet; if now the vertical shearing resistance is put as low as $\frac{1}{2}$ ton per square foot, each end would be credited with a power of resistance of 260 tons. The only way in which this resistance can be exerted is through the weight of the materials, added to the tensile 
strength of the mortar at the plane in question. But the Bouzey dam, above the level of the fissure, only weighed about 29 tons per lineal foot, and $\frac{1}{2}$ ton along the base would only be about 9 tons more per foot, so that the total vertical shearing resistance, which 1 foot in length, taken by itself, could exert, on the above assumption, is under 40 tons. The resistances, therefore, due to a length of nearly 7 feet, applied at the shearing line, would be required to balance a vertical shearing force of only $\frac{1}{2}$ ton per square foot. But those resistances are not available at that line. And, again, the horizontal shearing stresses, which could be exerted by the ends, would go a very small way in resisting the horizontal force of the water, accumulating over a long fissure; for the more the fissure progresses transversely, the more is the part of the dam above the fissure lifted from its seat and carried on a water-bearing, and at the same time, the more is the remaining sound portion at the plane of fissure reduced; so that the duty of resisting the horizontal force is more and more transferred from the base to the ends. It therefore appears that there is no proper shearing action, but rather a rending action, corresponding with a very low shearing stress, which would destroy in detail the dam at the line of fracture. No doubt the mortar in the Bouzey dam had some tensile strength, however low, and, if its value be assumed, it is possible to calculate the resistances required to be exerted by the ends. The moments, the sum of which must be zero, are those of the water in the fissure, the water in the reservoir, the weight of the dam, the shearing resistance of the ends, and the stresses in Fig. 3. The sum of the forces and stresses must also be zero. The stress of which $x$ is the vertical component is now equal to the tensile strength of the mortar, so that the value of $x$ is known. With these data, the value of the resistance of the ends, for any assumed value of $n$, can be found, but the calculation is somewhat long, and leads to no useful result, as in practice the resistance of the ends is, and ought to be, neglected. In any case, the Author believes that it is so small, where the fissure is long, compared with the other resistances and the forces, as not to be worth calculating.

The fissures to be next considered are those due to bad workmanship, occurring in dams of good design. It may be assumed that they are of limited extent, differing in this respect from those previously considered, which, being due to bad design, may extend for the whole length of the dam. The stresses in the case where the inner face of the dam is still in compression across the fissure are shown in Fig. 5 . 
The relations are-

$$
\begin{aligned}
\frac{1}{2} b^{2} x+\frac{2}{3} b^{2} \frac{y-x}{2}+\frac{1}{2} p n^{2} b^{2} & =m b \mathrm{~V} \\
b \frac{x+y}{2}+p n b & =\mathrm{V}
\end{aligned}
$$

therefore

$$
\begin{aligned}
& x=\frac{2 \mathrm{~V}}{b}(2-3 m)-p n(4-3 n) . . \\
& y=\frac{2 \mathrm{~V}}{b}(3 m-1)+p n(2-3 n) . .
\end{aligned}
$$

The ralue of the stress at any other point can be obtained directly from the Fig. when the values of $x$ and $y$ have been found.

Fig. 5.

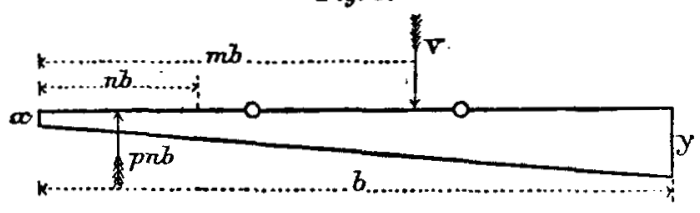

The case when $x=0$ may be specially noticed. In that case $p=\frac{2 \mathrm{~V}}{b} \cdot \frac{2-3 m}{n(4-3 n)}$. Now when there was no fissure, the value of Fig. 6.

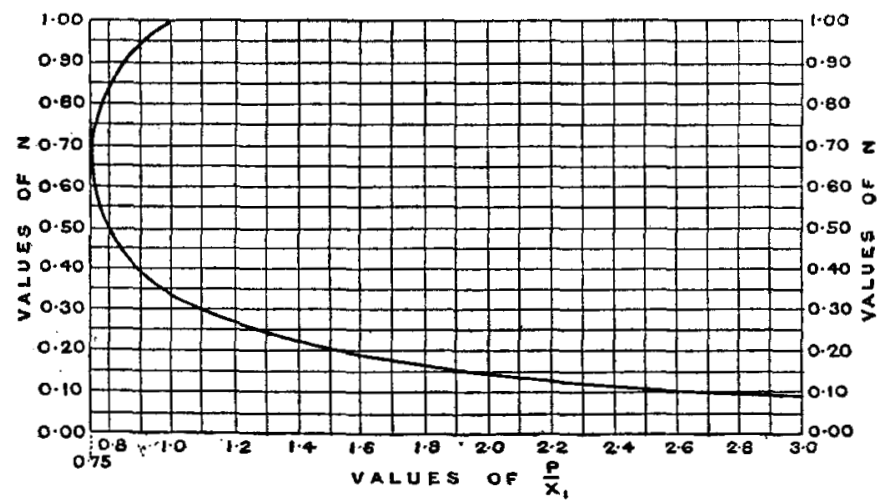

$x$ in (1) was $\frac{2 \mathrm{~V}}{b}(2-3 m)$; calling this, for the sake of distinction, $x_{1}$, for the case now considered

$$
\frac{p}{x_{1}}=\frac{1}{n(4-3 n)} \text {. . . . . }
$$


If the value of $\frac{p}{x_{1}}$ is greater than this quantity the fissure tends to open. The minimum value of the right hand side of $(10)$ is $\frac{3}{4}$, which occurs when $n=\frac{2}{3}$. If the value is less than $\frac{3}{4}$, the value of $x$ will not be zero; conversely, if the value of $n$ is greater or less than $\frac{2}{3}$, the value of the ratio must be greater than $\frac{3}{4}$ in order that $x$ may be zero. Again in (9) an increase in the value of $n$ causes an increase in the value of $y$ until $n=\frac{2}{3}$; any further increase in the value of $n$ causes the value of $y$ to decrease. The relations between $n$ and $\frac{p}{x_{1}}$ when $x=0$, are shown in Fig. 6 .

If the value of $\frac{p}{x_{1}}$ is greater than that in (10), $x$ will have a negative value in (8), but, as no tensile stresses can exist along the fissure, that equation fails, and the case is that treated uuder Fig. 7, the influence of the ends of the dam being neglected.

Fig. 7.

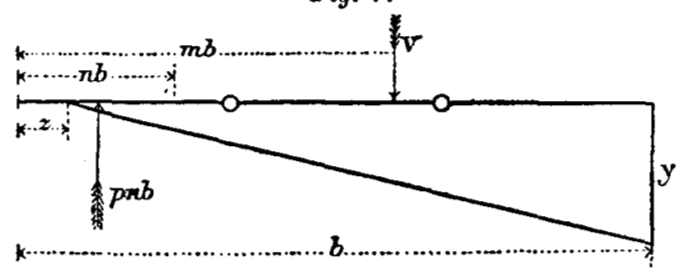

In this case the positive reactions along a part of the fissure have disappeared in consequence of the water-pressure in the fissure.

The relations are

$$
\frac{1}{2} y(b-z)\left(\frac{2 b+z}{3}\right)+\frac{1}{2} p n^{2} b^{2}=m b \mathrm{~V}
$$

therefore

$$
\frac{1}{2} y(b-z)+p n b=\mathrm{V}
$$

$$
\begin{aligned}
& z=\frac{b}{2(\mathrm{~V}-p n b)}\{2 \mathrm{~V}(3 m-2)+p n b(4-3 n)\} . \\
& y=\frac{1}{b} \cdot \frac{4(\mathrm{~V}-p n b)^{2}}{2 \mathrm{~V}(3 m-1)-p n b(2-3 n)} \cdot . . .
\end{aligned}
$$

The stress at any point can be obtained directly from the diagram when the values of $z$ and $y$ have been found.

$$
\text { When } z=n b \text {, }
$$

$$
y=\frac{2 V}{b(1-n)}-\frac{2 p n}{1-n} . \quad .
$$


When $p=\frac{2 \mathrm{~V}}{b} \cdot \frac{2-3 m+n}{n(4-n)}$, the stress in the sound part of the dam, immediately adjoining and behind the fissure, is reduced to zero. Then, taking the ratio as in. (10),

$$
\frac{p}{x_{\ell}}=\frac{2-3 m+n}{n(4-n)(2-3 m)} \cdot \text {. . . . }
$$

If $\frac{p}{x_{1}}$ has a valuo greater than in (14), the stress at the point referred to becomes negative.

In all these cases the value to be given to the ratio $\frac{p}{x_{1}}$ is of considerable importance in the upper part of a dam, as, if the dam at the top be too thin, the value of $\frac{p}{x_{1}}$ may, at certain levels, be too great for safety; this may be inferred from Fig. 6 .

In order to show the application of this investigation two numerical examples are given. Any support afforded by the ends of the dam is neglected. That support may hare, and in the case of short fissures unquestionably has, some value; but that value, depending on the size of the fissure, is uncertain, and no reliance can be placed upon it in any calculations for determining the stability of a dam. It was suggested above that such support is limited by the rending action to which the ends are liable which would seriously reduce their power of resistance below what it would be in the case of a simple shearing action.

In the first example let a horizontal plane be considered 100 feet below the top of the dam, with a maximum depth of water of 96 feet. Assume $V=217$ tons, $b=70$ feet, and $m=0 \cdot 593$. Then, by (1) and (2), $x=1 \cdot 37$ ton, and $y=4 \cdot 83$ tons. The value of $p$ in (10) is $2 \cdot 67$ tons, and the ratio $\frac{p}{x_{1}}=1 \cdot 95$. Now if a fissure be assumed, when in (10) $n=0 \cdot 144$, then $x=0$, and $y=5 \cdot 44$ tons. There is as jet no tendency on the part of the fissure to open. But if the fissure were, say, 20 feet deep, instead of 10 feet, the depth given by the value of $n$ last found, it is found from (8) and (9) that $x=-1 \cdot 02$ ton, and $y=5 \cdot 70$ tons. But, as no tensile stresses can exist across the fissure, the value of $y$ rises to that given by (12), viz., 6 tons, the value of $z$ in (11) being 15.38 feet. From (14), when $n=0.393$, that is, when the fissure extends from the face of the dam for a distance of 27.5 feet, the stress in the sound portion, immediately adjoining and behind the fissure, is reduced to zero, and from (13) $y=6 \cdot 76$ tons. For the purpose 
of this investigation, only the vertical component of the pressure is considered. For instance, the maximum unit stress, obtained by taking the resultant pressure acting on a plane at right angles to itself (a projection of a horizontal base) would, in the value of $y$ last given, be $9 \cdot 12$ tons instead of $6 \cdot 76$ tons.

For the second example a plane may be considered 150 feet below the top of the dam, with a depth of water of 146 feet. Assume $\mathrm{V}=536$ tons, $b=118$ feet, $m=0 \cdot 53$. Then by (1) and (2) $x=3 \cdot 72$ tons, and $y=5 \cdot 36$ tons, the corresponding maximum stresses being 4.86 tons and 7.01 tons respectively. The value of $p$ is $4 \cdot 07$, and that of $\frac{p}{x_{1}}$ in $(10)$ is therefore $1 \cdot 09$. Assuming a fissure as before, then in (10) when $n=0 \cdot 3$, that is, when the fissure extends from the inner face of the dam for a distance of 35.4 feet, then $x=0$, and in (9) $y=6.7$ tons. If now a greater value be taken for $n$, say $0 \cdot 4$, representing a depth of fissure of $47 \cdot 2$ feet, then from ( 8 ) and (9), $x=-0.8$ ton, and $y=6 \cdot 66$ tons. But as no tensile stresses can exist across the fissure the value of $y$ in (12) rises to $6 \cdot 8$ tons, the value of $z$ being $16 \cdot 87$ feet. This value of $y$ is equivalent to 8.89 tons maximum pressure. It is worth noticing that, with the value of $m$ assumed in this example, there is no value of $n$ which satisfies (14), so that the stress in the remaining sound part of the section, immediately adjoining and behind the fissure, is never reduced to zero, however long the fissure may be. The smallest value of $m$ which would have allowed such an effect is $0 \cdot 55$, and the value of $n$ would then have been 0.884 , giving a depth of fissure of $104 \cdot 3$ feet.

The values obtained in the preceding examples are the result of considering special cases, and depend upon what may be called the characteristic values of $\mathrm{V}, b$ and $\mathrm{m}$. Still, they are sufficient to show that with a well-designed profile it is possible to overestimate the detrimental effect of a fissure if the subject bo approached, as it usually is, from a statical point of view; and, from this point of view, honeycombing of the material, allowing percolation of water, would be less detrimental than a fissure of equal area; and this is perhaps more likely to occur as the result of bad workmanship than an absolute fissure, although it does not require very great carelessness in joining up work to create a fissure. These remarks apply only to those cases in which the fissure has not been originated by the water-pressure acting on a dam of faulty design; and it is also assumed that there is some margin in the profile, beyond the net area required, to allow for contingencies of every sort. This is only saying 
that the dam must have a good ordinary profile as now understood, in which the value of $m$ does not too nearly approach twothirds. Lastly, the thickness of the dam at the top must not be so small that the value of $\frac{p}{x_{1}}$ becomes at any level unduly large. In neither of the two numerical examples taken was any dangerous result arrived at, though the depth of the fissures assumed was considerable, and far exceeded anything likely to occur in practice.

The following Table shows all the characteristic quantities for the Periyar dam on the assumption that the weight of the masonry and concrete is equivalent to an average of $150 \mathrm{lbs}$. per cubic foot. The last two columns show the depth of fissure necessary to reduce $x_{1}$ to zero in equation (10), the values of $n$ being taken from Fig. 6 .

\begin{tabular}{|c|c|c|c|c|c|c|c|c|}
\hline \multirow{2}{*}{$\begin{array}{c}\text { Level } \\
\text { T.W.L. } \\
=155 \text { Feet. }\end{array}$} & \multirow{2}{*}{ V. } & \multirow{2}{*}{$b}$. & \multirow{2}{*}{$m$} & \multirow{2}{*}{$x_{1}$. } & \multirow{2}{*}{$p$} & \multirow{2}{*}{$\frac{p}{x_{1}}$} & \multicolumn{2}{|c|}{$x_{1}=0$ when } \\
\hline & & & & & & & $n$. & $n b$. \\
\hline Feet. & Tons. & Feet. & $\begin{array}{l}\text { Tons per } \\
\text { sq Fuot. }\end{array}$ & $\begin{array}{l}\text { Tons per } \\
\text { Sq. Foot. }\end{array}$ & $\begin{array}{l}\text { Tons per } \\
\text { Sq. Foot. }\end{array}$ & $\begin{array}{l}\text { Tons per } \\
\text { Sq. Foot. }\end{array}$ & $\begin{array}{l}\text { Tons per } \\
\text { Sq. Foot. }\end{array}$ & Feet. \\
\hline 145 & $10 \cdot 38$ & $12 \cdot 00$ & $0 \cdot 544$ & $0 \cdot 637$ & $0 \cdot 279$ & 0.438 & & $\because \ddot{0}$ \\
\hline 13 & $19 \cdot 09$ & $14 \cdot 00$ & $0 \cdot 588$ & 0.644 & 0.557 & 0.865 & 0.424 & $5 \cdot 936$ \\
\hline 12 & $29 \cdot 80$ & $18 \cdot 00$ & 0.618 & 0.483 & 0.836 & $1 \cdot 731$ & 0.168 & $3 \cdot 024$ \\
\hline 115 & $43 \cdot 86$ & $24 \cdot 00$ & $0 \cdot 619$ & 0.523 & $1 \cdot 114$ & $2 \cdot 130$ & $0 \cdot 129$ & $3 \cdot 096$ \\
\hline 105 & $62 \cdot 27$ & $30 \cdot 6$ & 0.625 & 0.509 & $1 \cdot 393$ & $2 \cdot 737$ & 0.099 & $3 \cdot 029$ \\
\hline 95 & $85 \cdot 12$ & $37 \cdot 20$ & 0.634 & 0.448 & $1 \cdot 672$ & $3 \cdot 732$ & 0.070 & $2 \cdot 604$ \\
\hline 85 & $112 \cdot 57$ & $43 \cdot 87$ & $0 \cdot 641$ & $0 \cdot 395$ & $1 \cdot 950$ & $4 \cdot 937$ & 0.053 & $2 \cdot 325$ \\
\hline 75 & $144 \cdot 78$ & $50 \cdot 64$ & 0.645 & $0 \cdot 372$ & $2 \cdot 229$ & $5 \cdot 992$ & 0.043 & $2 \cdot 188$ \\
\hline 65 & $182 \cdot 25$ & $58 \cdot$ & $0 \cdot 6$ & 0.536 & $2 \cdot 507$ & $4 \cdot 677$ & $0 \cdot 056$ & $3 \cdot 278$ \\
\hline 55 & $226 \cdot 58$ & $69 \cdot 57$ & $0 \cdot 606$ & $1 \cdot 186$ & $2 \cdot 786$ & $2 \cdot 349$ & $0 \cdot 117$ & $8 \cdot 140$ \\
\hline 45 & $279 \cdot 06$ & $80 \cdot 80$ & $0 \cdot 584$ & $1 \cdot 713$ & $3 \cdot 065$ & $1 \cdot 789$ & $0 \cdot 159$ & $12 \cdot 847$ \\
\hline 35 & 340.08 & $92 \cdot 26$ & 0.568 & $2 \cdot 182$ & $3 \cdot 343$ & $1 \cdot 532$ & $0 \cdot 191$ & $17 \cdot 622$ \\
\hline 25 & $410 \cdot 07$ & 106.99 & 0.539 & $2 \cdot 936$ & $3 \cdot 622$ & $1 \cdot 234$ & $0 \cdot 250$ & $26 \cdot 747$ \\
\hline 15 & $496 \cdot 09$ & $117 \cdot 49$ & 0.544 & $3 \cdot 108$ & $3 \cdot 900$ & $1 \cdot 255$ & $0 \cdot 244$ & $28 \cdot 668$ \\
\hline 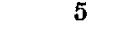 & $578 \cdot 29$ & $128 \cdot 00$ & 0.536 & $3 \cdot 542$ & $4 \cdot 179$ & $1 \cdot 180$ & $0 \cdot 265$ & $33 \cdot 920$ \\
\hline
\end{tabular}

From this point of view the level at which the minimum strength of the dam occurs is 75 feet above datum, or 80 feet below top-water level. Concentrating attention upon the conditions at this level, and referring to the remarks upon equation (6), it is found that the quantity $\overline{2 a+3 r}=2 \cdot 139,>2$; consequently, the negative value of $x$ (the stress at the inner extremity of the fissure) in (6) increases when the value of $n$ is increased. Then in (6) $x=0$ when $n=0 \cdot 127$, the depth of the fissure being 6.43 feet; this result may also be obtained from (14). When the negative or tensile value of $x$ in (6) is as great as, say, 1 ton per square foot, the value of $n$ is 0.3326 , and the depth of the fissure 
is 16.84 feet. If, however, the weight of the masonry and concrete were only 135 lbs. per cubic foot, the value of $m$ at the level under consideration rises to 0.6811 , and the inner face is therefore in tension, the value of $x_{1}$ being -0.223 ton. If the top of the profile were cut down, in order to form an overflow weir, the result would be still less favourable. The value of $\overline{2} \overline{a+3 r}=3 \cdot 042,>2$, and therefore, if there be a fissure, any increase in the depth of that fissure is accompanied by an increase in the tensile stress at its inner extremity. It is therefore clear that this profile, though proper for a dam in which, as at Periyar, the materials have a fairly high specific gravity, would not be satisfactory where that is not the case; and it is to be remembered that the weight of $135 \mathrm{lbs}$. per cubic foot is not a minimum weight, for that at Bouzey was only 125 lbs. per cubic foot. This instance is sufficient to show that careful consideration is necessary, before accepting any formula for determining the profile of a dam, in which the result is independent of the specific gravity of the materials. The quantity $\frac{p}{x_{1}}$, regarded as a sort of fourth dimension in the process of designing a dam, affords an exact measure of the margin of safety against imperfect workmanship provided in any particular case.

The foundations of a daw are (or ought to be) always carried some distance into solid rock; when that is the case, the rock sides against which the masonry is flushed, hold that masonry so that it may be considered a part of the rock rather than of the dam proper ; and for this reason and for reasons already stated, it is, in the Author's opinion, unnecessary to consider the lifting action of any water which may be present in the rock beneath the foundations, for any circumstances likely to occur in practice, although, if these limitations be not taken into account, alarming conditions can no doubt be arrived at by calculation.

This is not the case, however, with vertical fissures, caused by the contraction of the material when the temperature is lower than that obtaining when the work was constructed. Pure theory and fact in this case go hand in hand; there are no natural limitations in practice, neither have any yet been devised. It has indeed been suggested that, if the dam be curved in plan, it is free to expand and contract without unduly stressing the masonry; if this is true in some cases, it is a fact that it is not so in others. If the data, such as the tensile strength, modulus of elasticity, and coefficient of expansion of the masonry be known, and theory predicts temperature cracks, those cracks will occur. Attention 
has, not infrequently, been directed to the possibility of such cracks occurring, but the Author believes that there is a general impression that this possibility is so remote that it need not be considered. When these cracks occur, they begin at the exposed face, and extend into the work, gradually disappearing at a distance from the face corresponding to the penetration of the low temperature. If the work be comparatively thin, and the temperature very low, the cracks may extend from one face to the other. Masonry dams, besides being more exposed to variation of temperature than ordinary retaining walls, have this further disadvantage, that their ends, by being carried into the rock, are fixed, and therefore the length of the surfaces exposed to variation of temperature is also fixed. It appears that in some cases nutation of the top of the dam has been observed, corresponding to some extent with the variation of temperature, and possibly to some extent relieving the temperature stresses. This can perhaps be understood if it takes place when the variation of temperature, whether above or below the normal, on both sides of the dam, is not the same. It is, however, difficult to see how nutation can take place, at any rate in a dam, of which the line is straight, when this is not the case. It does not, however, appear probable that, even if the dam be curved, the stresses can be relieved to any considerable degree, as the variation in temperature which produces cracks is a local variation; it is not uniform throughout the width of the dam, neither does it vary uniformly from one side to the other. Cracks of this nature cannot be avoided even in work of first-class quality; only in that case they may pass impartially through stone and mortar, instead of following the joints as when the mortar is weak. It is fortunate that they do not, by themselves, in any way reduce the stability of a dam, but, unless these facts are realized, their appearance is certain to cause disappointment.

The Paper is accompanied by two tracings, from which the Figs. in the text have been prepared. 